\title{
2022 Update of BCLC Treatment Algorithm of HCC: What's New for Interventional Radiologists?
}

\author{
Pierleone Lucatelli ${ }^{1} \cdot$ Boris Guiu ${ }^{2}$ (D)
}

Received: 4 December 2021 / Accepted: 12 December 2021/Published online: 28 January 2022

(C) Springer Science+Business Media, LLC, part of Springer Nature and the Cardiovascular and Interventional Radiological Society of Europe (CIRSE) 2021

The Barcelona Clinic Liver Cancer (BCLC) system, initially proposed in 1999, is certainly the most commonly used staging system for HCC in Western countries. Just a few days ago, the BCLC group released the 2022 update published in the Journal of Hepatology [1]. This latest version turns the previous rigid BCLC staging system toolbox into a flexible and versatile instrument, with which interventional radiologists (IRs) can play an even more central role.

One major novelty in this update relates to the endorsement of treatment stage migration (TSM). The 2022 BCLC strategy incorporates an expert clinical decision-making component, formally permitting tailored treatment on the basis not only of the patient's and tumor's characteristics but also of local expertise and technical availability. TSM is applied when a specific patient profile or treatment failure/unfeasibility may induce a shift of the recommendation to the option that would be considered for a more advanced stage. In a 2021 issue of CVIR, deviation from BCLC first-treatment recommendation resulting from a multidisciplinary, individualized approach actually showed OS yielding or exceeding expected survival at each BCLC stage [2].

The second novelty in this update is the recognition of liver transplantation (LT) as one of the main objectives. Compared to the 2018 version where LT was (only) recommended in multifocal $\leq 3 \mathrm{~cm}$ HCCs, three arrows are now directed toward LT, one from each of the following: small multifocal HCCs, a subgroup of BCLC B patients, in case of successful downstaging by TACE or TARE. The role of IRs now becomes central within MDT to reach the goal of increasing the number of transplanted patients.

As a consequence, these novelties lead to a more complex BCLC algorithm. To improve clarity, we aimed to summarize below the role of IRs at each BCLC stage.

In BCLC 0, ablation is the preferred option. However if it is not feasible for any reason, resection should be considered first and then TACE, in keeping with the concept of stage migration. TARE is considered as effective as TACE, but is recommended only in single HCC $\leq 8 \mathrm{~cm}$, given the results of the LEGACY trial [3].

In BCLC A, resection is favored in $>2 \mathrm{~cm}$ HCC because of higher recurrence rate after ablation. In large tumors, radiation lobectomy by TARE is not explicitly recommended but can be considered in case of small future liver remnant. In non-LT candidates with multifocal tumors, the 2022 BCLC update does not recommend resection but rather ablation for HCCs $\leq 3 \mathrm{~cm}$ and TACE otherwise. TARE might be indicated when meeting inclusion criteria of LEGACY. In LT candidates with $>6$ months waiting time, ablation, TACE or TARE can be used for bridging.

The 2022 BCLC version stratifies the BCLC-B into three groups of patients according to tumor burden and liver function. The first subgroup corresponds to patients candidates for LT if they meet the 'Extended Liver Transplant criteria'

Boris Guiu

B-guiu@chu-montpellier.fr

1 Department of Radiology, Vascular and Interventional Radiology Unit, Sapienza University of Rome, Rome, Italy

2 Department of Radiology, St-Eloi University Hospital, 80 avenue Augustin Fliche, 34295 Montpellier, France 
(usually based on size and/or AFP) endorsed by each institution/country. The authors acknowledge the potential role of downstaging to LT [4] through liver-directed therapies but call for further studies on how to best apply a downstaging protocol. The second subgroup is composed by patients without option for LT but who have preserved portal flow and welldefined nodules allowing selective access to feeding tumor arteries. They are (the only) candidates for TACE. The third subgroup includes patients with diffuse, infiltrative, extensive bilobar liver involvement. Systemic treatment is recommended for these patients who basically do not benefit from TACE. Categorization of patients in this third subgroup remains, however, subjective, since no cutoff is provided. Of note, patients with $>2 \mathrm{mg}$ bilirubin level or slight fluid retention (even on imaging) requiring diuretic treatment are also considered poor candidates for TACE. TACE type (5) (conventional or using drug-eluting microsphere) is left to local discretion. Overall, the place of TACE in BCLC B is somewhat restricted and TARE is not even mentioned.

Finally, in contrast both to real-life practice and to the most recent results of TARE in advanced HCC, the 2022 update does not recognize any role to IRs in BCLC C patients because of negative phase III trials.

In conclusion, this update clarifies the role of IRs across BCLC 0/A/B stages. As expected, multi-directional treatment allocation and TMS make the algorithm more complex to read, but they bring it closer to daily practice. The very limited role lent to TARE might be disappointing, but it is already a big step forward compared to before. This highlights more than ever that there is still a lot of work to reach level one evidence, but it seems definitely within our reach!

Funding None.

\section{Declarations}

Conflicts of interest Boris Guiu is a consultant for Boston Scientific, Guerbet, Terumo and Quantum Surgical, received honorarium (outside this work) from Boston Scientific, Terumo, Quantum Surgical, Ipsen and Roche and received study funding (outside this work) from Boston Scientific, Guerbet and Roche. Pierleone Lucatelli is a consultant for Terumo. He has received honorarium from Guerbet (outside this work).

Ethical Approval This article does not contain any studies with human participants or animals performed by any of the authors.

Informed Consent For this type of study, informed consent is not required.

Consent for Publication For this type of study, consent for publication is not required.

\section{References}

1. Reig M, Forner A, Rimola J, Ferrer-Fábrega J, Burrel M, Garcia-Criado A, et al. BCLC strategy for prognosis prediction and treatment recommendation Barcelona Clinic Liver Cancer (BCLC) staging system. The 2022 update. J Hepatol. 2021. https://doi.org/10.1016/j.jhep. 2021.11.018.

2. Matsumoto MM, Mouli S, Saxena P, Gabr A, Riaz A, Kulik L, et al. Comparing real world, personalized, multidisciplinary tumor board recommendations with BCLC algorithm: 321-patient analysis. Cardiovasc Intervent Radiol. 2021;44(7):1070-80.

3. Salem R, Johnson GE, Kim E, Riaz A, Bishay V, Boucher E, et al. yttrium-90 radioembolization for the treatment of solitary unresectable HCC: the LEGACY study. Hepatology. 2021;74(5):2342-52.

4. Mazzaferro V, Citterio D, Bhoori S, Bongini M, Miceli R, De Carlis L, et al. Liver transplantation in hepatocellular carcinoma after tumour downstaging (XXL): a randomised, controlled, phase 2b/3 trial. Lancet Oncol. 2020;21(7):947-56.

5. Lucatelli P, Burrel M, Guiu B, de Rubeis G, van Delden O, Helmberger T. CIRSE standards of practice on hepatic transarterial chemoembolisation. Cardiovasc Intervent Radiol. 2021;44(12):1851-67.

Publisher's Note Springer Nature remains neutral with regard to jurisdictional claims in published maps and institutional affiliations. 